\title{
The hospital foodscape: characterisation of the hospital food fringe across London
}

It is estimated that 700,000 of the 1.2 million NHS staff are overweight or obese ${ }^{(1)}$. This is recognised as a significant contributor to employee illness and absence with an annual cost to the NHS of $£ 2.4$ billion. As part of the NHS Five Year Forward View ${ }^{(2)}$, there is an aim to create a healthier food environment for staff. Study of the environment in which staff make food choices must extend to the external environment, the hospital food fringe (HFF). There is growing pressure on Local Authorities to improve the food environment through planning including reducing the clustering of fast food outlets (FFO) around schools and other facilities for children and families $^{(3)}$. The aim of this study was to describe food outlet provision (frequency and type) within the HFF of twenty London hospitals.

A purposive sample of London hospitals were included in the study. Digimap Ordnance Survey software ${ }^{(4)}$ was used to produce a 1:5000 scale map of the HFF, a 400 m radius from main entrance of the hospital. Groundtruthing (direct observation) was used to characterize outlet density ${ }^{(5)}$ and an adapted Food Outlet Classification tool was used to classify outlet typologies ${ }^{(6)}$. Area level deprivation was assessed using the Index of Multiple Deprivation (IMD). Statistical analysis was conducted using SPSS 24 with statistical significance observed at $\mathrm{p}=0.05$.

Twenty London hospitals, $75 \%$ inner London; average bed size 507 (range 100-1000) were assessed. The median number of food outlets per hospital was 21.5 (range $0-87$ ) of which $15.3 \%$ were FFO with $75 \%$ of hospitals having at least one FFO within a $400 \mathrm{~m}$ radius. No association between total outlet $(\mathrm{r}=0.182, \mathrm{p}=0.443)$ or FFO density $(\mathrm{r}=-0.260, \mathrm{p}=0.268)$ and deprivation was observed. As expected, inner London hospitals had greater total outlet $(p=0.015)$ and FFO $(p=0.041)$ density compared to outer London hospitals.

A retail foodscape that supports healthy food choice can be characterised as one which restricts opportunities to purchase energydense foods of low nutritional value and provides abundant opportunity to purchase healthier foods ${ }^{(7)}$. The composition of the HFF varied enormously between hospitals with inner city hospitals having a greater density of total food outlets and FFO. Creating a healthy food environment will require organisations to develop a strategy based on analysis of the drivers and barriers its staff encounter. Characterisation of the hospital foodscape is a necessary first step and this study informs a robust methodology that can be used in local policy making.

1. Healthy weight, healthy lives: One year on (2009) Available at: http://bit.ly/2Healthyweighthealthylives2009

2. NHS Five Year Forward View: NHS England (2014) Available at: http://bit.ly/2FiveYearForwardView

3. Health matters: obesity and the food environment: Public Health England (2017) Available at: http://bit.ly/2HealthMattersObesityEnvironment

4. EDINA Digimap Ordance Survey Service (2015) Available from: https://digimap.edina.ac.uk/

5. Rossen LM, Pollack KM, \& Curriero FC (2012) Health \& Place 18, 956-62.

6. Lake AA, Burgoine T, Stamp E et al. (2012) Int J Behav Nutr Phys Act 9, 37-49.

7. Swinburn B, Vandevijvere S, Kraak V et al. (2013) Obes Rev 14, 24-37. 